\title{
Metastatic Nasopharyngeal Carcinoma Simulating a Schwannoma of the Vagus Nerve
}

\author{
Maria Pascual-Gallego, Angela Moreno-Gutierrez, Raquel Gutierrez-Gonzalez, Gregorio Rodriguez-Boto
}

\begin{abstract}
Schwannomas of the vagus nerve are extremely rare neoplasms of difficult diagnosis and with a challenging surgical excision. We report a patient with a parapharyngeal mass radiologically indistinguishable from a schwannoma that was finally diagnosed as metastatic nasopharyngeal carcinoma.
\end{abstract}

Keywords: Metastasis, Nasopharyngeal carcinoma, Nasopharyngeal, Cancer, Parapharyngeal, Tumor.

How to cite this article: Pascual-Gallego M, MorenoGutierrez A, Gutierrez-Gonzalez R, Rodriguez-Boto G. Metastatic Nasopharyngeal Carcinoma Simulating a Schwannoma of the Vagus Nerve. Int J Head and Neck Surg 2013;4(1):44-46.

\section{Source of support: Nil}

\section{Conflict of interest: None}

\section{INTRODUCTION}

Tumors of the parapharyngeal space are uncommon, comprising less than $1 \%$ of all head and neck neoplasms. Differential diagnosis includes salivary gland neoplasms, nerve sheath tumors and other miscellaneous lesions. Most tumors in this space are metastatic lesions or direct extensions from adjacent spaces. Nerve sheath tumors may arise from any of the cranial nerves in the region (cranial nerves IX, X, XII), as well as from the cervical sympathetic chain.

We describe an exceptional case of metastatic nerve sheath tumor arising from the vagus nerve and corresponding to a nondiagnosed undifferentiated nasopharyngeal carcinoma (NPC) that radiologically simulated a vagal schwannoma.

\section{CASE REPORT}

A 63-year-old Caucasian male with personal history of transient ischemic attack and ex-smoker was referred for an enlarging, painless left neck mass that occasionally provoked sensory disturbance around it. No neurological disorder was observed after neurological examination.

Magnetic resonance imaging (MRI) of the neck showed a well-circumscribed solid lesion, $2.5 \times 2 \times 2 \mathrm{~cm}$ in size and located in the left carotid space. The mass was isointense in T1-weighted sequences and hypointense in T2-weighted sequences. It also presented slightly isointense cystic areas on the upper pole and showed uniform enhancement after contrast administration, suggesting schwannoma of the vagus nerve. Carotid body tumor was ruled out by cervical angiography (Figs 1A and B). A left transcervical approach was performed demonstrating a solid tumor adhered to the vagus nerve. Gross total resection of the lesion was accomplished (Figs 2A to C). Despite the absence of MRI findings for that specific zone, histological study revealed that the lesion was a lymph node infiltrated by an undifferentiated NPC (Figs 3A and B). In the postoperative course, the patient received radiotherapy and chemotherapy and after 4 years follow-up, remains asymptomatic.
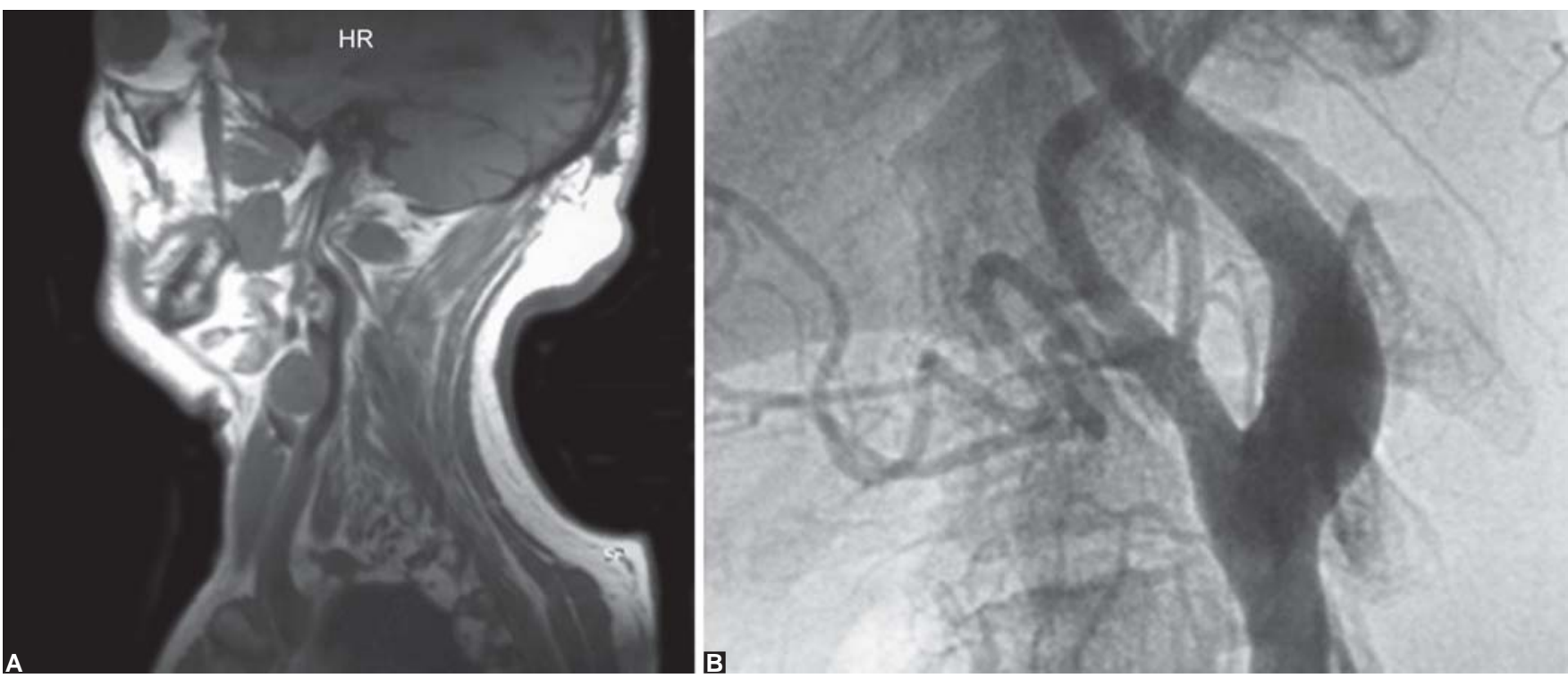

Figs 1A and B: (A) Sagittal T1-weighted MRI showing a $2.5 \times 2 \times 2 \mathrm{~cm}$ lesion in the left carotid space, (B) cervical angiography ruled out a vascular lesion 

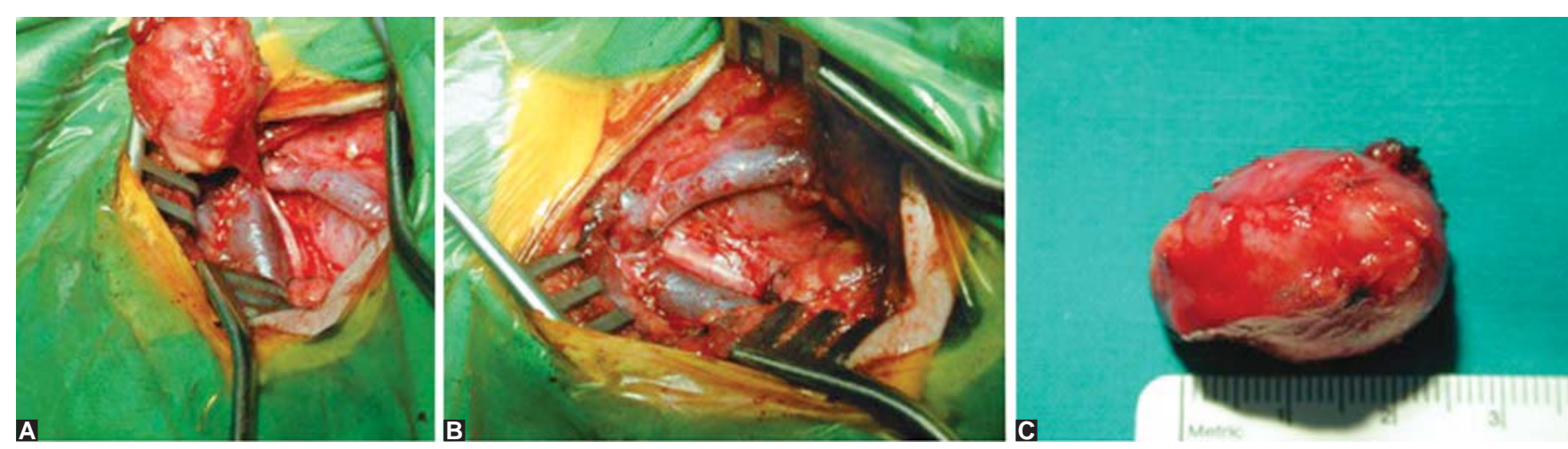

Figs 2A to C: (A) Intraoperative images of the lesion. A well-circumscribed solid lesion is identified adhered to the vagus nerve between the common carotid artery and the internal jugular vein, (B) gross total resection of the tumor, (C) anatomic piece $2.5 \times 2 \times 2 \mathrm{~cm}$ in size
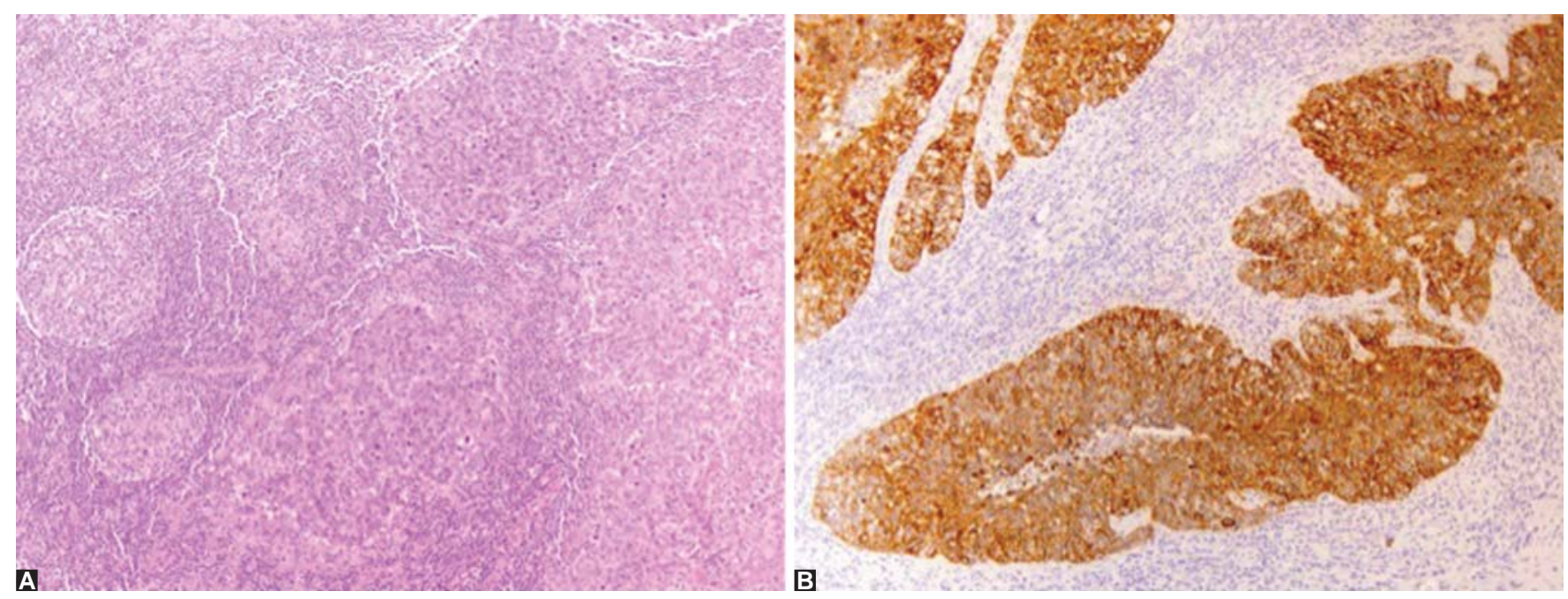

Figs $3 A$ and B: (A) Histopathology showing partial replacement of lymph node by epithelial neoplastic cell nests (H\&E staining, $\times 200)$, (B) immunohistochemistry showing positivity to cytokeratins AE1/AE3 (×200)

\section{DISCUSSION}

Nasopharyngeal carcinoma incidence varies according to geographic location. Incidence is approximately 0.5 to $1 / 100,000$ people annually in Europe, which accounts for 2 to $3 \%$ of all head and neck tumors. ${ }^{1}$ Patients are usually diagnosed in advanced states, and although rhinological symptoms are more frequent, cervical adenopathies are the main reason for consultation. ${ }^{2}$

Carcinomas account for $85 \%$ of nasopharyngeal malignant lesions and constitute the most frequent histological subtype of NPCs. The undifferentiated type is the most frequent one and has a more favorable outcome than differentiated types. ${ }^{3}$

MRI is now the preferred imaging modality in the assessment and staging of NPC, in relation with its superior soft tissue contrast, the ability to demonstrate perineural tumor spread and bone narrow involvement as well as its ability to show the involvement of adjacent structures. Differential diagnosis of parapharyngeal-space neoplasm's includes salivary gland neoplasms, nerve sheath tumors and other lesions including carotid body tumor, abscess, branchial cleft cyst, malignant lymphoma or metastatic cervical lymphadenopathy.,

Radiotherapy constitutes the treatment of choice whereas surgery is usually limited to biopsy. However, in cases of advanced states, adjuvant chemotherapy may also be considered after radiotherapy. ${ }^{6}$ Distant metastases constitute the main impact factor on survival, reported to be 30 to $65 \%$ in 5 years. ${ }^{1}$

Up to 20 to $27 \%$ of all the tumors that can be found at the parapharyngeal space are benign, being among them the schwannoma the most common one. ${ }^{7}$ Besides that, schwannomas of the vagus nerve and the cervical sympathetic chain are the most commonly reported.

Diagnosis is achieved by means of MRI studies, where the schwannomas are shown generally as solitary wellcircumscribed solid lesions, isointense in T1-weighted sequences, that show uniform enhancement after contrast administration. An accurate differential diagnosis of other kind of lesions also present in the parapharyngeal space is essential in order to predict the different complications that 
may arise from the tumor itself, as well as to determine if intraoperative nerve monitoring is required.

Surgery is the treatment of choice for parapharyngeal space tumors. Surgical approaches are dictated by the size of the lesion, the location, the anatomical relationship with the great vessels and the suspicion of malignancy. 8,9

\section{CONCLUSION}

We describe an exceptional case of metastatic nerve sheath tumor arising from the vagus nerve. It is estimated that approximately 3 to $5 \%$ of the primary tumor that origins this type of lesions is never identified, as occurred in our case. ${ }^{10}$

\section{REFERENCES}

1. Liu MT, Hsieh CY, Chang TH, Lin JP, Huang CC, Wang AY. Prognostic factors affecting the outcome of nasopharyngeal carcinoma. Jpn J Clin Oncol 2003;33(10):501-08.

2. Sang J, Lou W, Zhang Y. Diagnosis and surgical approach of parapharyngeal space neoplasms. Lin Chung Er Bi Yan Hou Tou Jing Wai Ke Za Zhi 2011;25(21):961-65.

3. Shanmugaratnam K, Sobin LH. The World Health Organization histological classification of tumours of the upper respiratory tract and ear. A commentary on the second edition. Cancer 1993; 71(8):2689-97.

4. Goh J, Lim K. Imaging of nasopharyngeal carcinoma. Ann Acad Med Singapore 2009;38(9):809-16.

5. Anil G, Tan TY. CT and MRI evaluation of nerve sheath tumors of the cervical vagus nerve. Am J Roentgenol 2011;197(1): 195-201.

6. Setton J, Wolden S, Caria N, Lee N. Definitive treatment of metastatic nasopharyngeal carcinoma: Report of 5 cases with review of literature. Head Neck 2012;34(5):753-57.
7. Dimitrijevic MV, Jesic SD, Mikic AA, Arsovic NA, Tomanovic NR. Parapharyngeal space tumors: 61 case reviews. Int J Oral Maxillofac Surg 2010:39(19):983-89.

8. Papadogeorgakis N, Petsinis V, Goutzanis L, Kostakis G, Alexandridis C. Parapharyngeal space tumors: Surgical approaches in a series of 13 cases. Int J Oral Maxillofac Surg 2010;39(3):243-50.

9. Zhi K, Ren W, Zhou H, Wen Y, Zhang Y. Management of parapharyngeal-space tumors. J Oral Maxillofac Surg 2009; 67(6):1239-44.

10. Ichikawa Y, Okamoto I, Nakagawa K. Cancer of unknown primary site-current status and future direction. Gan To Kagaku Ryoho 2009;36(6):907-09.

\section{ABOUT THE AUTHORS}

\section{Maria Pascual-Gallego (Corresponding Author)}

Department of Neurosurgery, Hospital Clínico San Carlos Universidad Complutense de Madrid, Madrid, Spain, e-mail: mariapascualgallego@gmail.com

\section{Angela Moreno-Gutierrez}

Department of Neurosurgery, Hospital Clínico San Carlos, Universidad Complutense de Madrid, Madrid, Spain

\section{Raquel Gutierrez-Gonzalez}

Department of Neurosurgery, Hospital Clínico San Carlos, Universidad Complutense de Madrid, Madrid, Spain

\section{Gregorio Rodriguez-Boto}

Department of Neurosurgery, Hospital Clínico San Carlos, Universidad Complutense de Madrid, Madrid, Spain 